\title{
Effacement énonciatif et doxa dans le discours théorique : l'exemple de Julia Kristeva
}

The erasement of subjectivity and doxa in theoretical discourse: the case of Julia Kristeva

\section{François Provenzano}

\section{(2) OpenEdition \\ Journals}

Édition électronique

URL : http://journals.openedition.org/aad/973

DOI : $10.4000 /$ aad. 973

ISSN : 1565-8961

Éditeur

Université de Tel-Aviv

\section{Référence électronique}

François Provenzano, «Effacement énonciatif et doxa dans le discours théorique : l'exemple de Julia Kristeva », Argumentation et Analyse du Discours [En ligne], 5 | 2010, mis en ligne le 20 octobre 2010, consulté le 23 septembre 2019. URL : http://journals.openedition.org/aad/973 ; DOI : 10.4000/aad. 973

Ce document a été généré automatiquement le 23 septembre 2019.

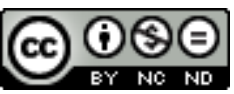

Argumentation \& analyse du discours est mis à disposition selon les termes de la licence Creative Commons Attribution - Pas d'Utilisation Commerciale - Pas de Modification 4.0 International. 


\section{Effacement énonciatif et doxa dans le discours théorique : l'exemple de Julia Kristeva}

The erasement of subjectivity and doxa in theoretical discourse: the case of Julia Kristeva

François Provenzano

1 Le présent article entend embrasser la problématique de l'effacement énonciatif dans le discours théorique et, à partir d'une étude de cas centrée sur le discours de Julia Kristeva, ébaucher quelques réflexions de méthode quant à l'articulation des plans sociologiques et discursifs de l'analyse ${ }^{1}$.

\section{L'effacement énonciatif : bref état de la question}

2 Les travaux de Catherine Kerbrat-Orecchioni sur la subjectivité dans le langage ont déjà, de longue date, judicieusement attiré l'attention des linguistes sur la complexité des mécanismes énonciatifs. Trois constats essentiels nous semblent devoir être rappelés ici : celui de "l'impossibilité de l'objectivité discursive» (Kerbrat-Orecchioni 2009 : 146), celui de la «dynamique argumentative » dans laquelle certains signifiants trouvent leur pertinence énonciative (ibid.), enfin celui de la manifestation graduelle de la subjectivité dans le langage (ibid.: 174), dont les marques se déclinent des plus explicites (comme les déictiques) aux plus implicites (comme les opérations de sélection et de hiérarchisation de l'information ou le mode de donation des référents).

Chacun de ces constats a ouvert la voie à d'importants chantiers de recherche qui, plutôt que de traquer les interventions subjectives dans les énoncés, ont privilégié l'enquête sur les procédés linguistiques producteurs de ce que Kerbrat-Orecchioni avait, dès 1978, appelé "l'effet d'objectivité $»^{2}$, autrement dit sur la manière dont l'énonciateur agence son propre effacement énonciatif. Robert Vion a insisté sur le caractère stratégique que revêt l'usage de tels procédés, qui visent à «construire un 
énonciateur "universel" [...] porteur de la raison et de l'objectivité " (Vion 2001 : 334-35). Il associait également ce type de stratégie au discours scientifique et théorique, mais aussi au discours publicitaire, juridique ou journalistique.

Gilles Philippe a quant à lui radicalisé la démarche, en émettant l'hypothèse d'un « appareil formel de l'effacement énonciatif » qui, mis en œuvre dans certains énoncés aux contrats de lecture spécifiques, peut produire un «ethos textuel»: «les textes seront jugés austères ou plaisants, élégants, sérieux ou pessimistes, sans que ces qualités soient forcément rapportées à une figure de locuteur» (Philippe $2002: 28$ ). Enfin, cette conception "positive » de l'effacement énonciatif et cette attention aux effets de lecture qu'il entraîne sont aussi celles d'Alain Rabatel, qui propose en outre d'associer ce type de configuration énonciative à ce que Ruth Amossy a appelé la « dimension argumentative » d'un discours (Amossy 2006 [2000]). À partir d'un texte de Semprun, Rabatel montre en effet que « les énoncés qui effacent l'origine énonciative $\mathrm{du}$ locuteur comportent des contenus plus directement acceptables pour le coénonciateur que ceux qui passent par la médiation du locuteur-énonciateur »; ces énoncés impliquent un « surcroît de travail interprétatif » qui consolide l'adhésion à la thèse défendue (Rabatel 2004 : § 58).

De ce parcours au pas de charge, nous retiendrons a) que l'effacement énonciatif est un simulacre linguistique, relevant d'une stratégie énonciative prise dans un continuum entre la subjectivation et l'effet d'objectivité ; b) que ce type de stratégie est propre à certains genres de discours dans lesquels le locuteur a intérêt à afficher un ethos de neutralité et d'objectivité; c) que cet ethos apparent dissimule souvent des effets argumentatifs plus complexes, dont les enjeux sont interactionnels, interprétatifs, voire éthiques (Koren 1996).

\section{Le discours théorique : énonciation, doxa, contrat rhétorique}

Quelle place occupe le cas du discours théorique dans ce cadre problématique? Il constitue sans aucun doute l'un des lieux privilégiés des stratégies d'effacement énonciatif, dans la mesure où l'ethos d'objectivité relève du contrat rhétorique propre à ce type de productions discursives : le lecteur de théorie s'attend à ce que l'énonciateur d'un tel discours donne l'impression qu'une vérité intemporelle se déploie, à propos de tel ou tel objet traité par la théorie, indépendamment de toute évaluation subjective et de tout ancrage spatio-temporel.

7 Cela dit, paradoxalement, l'énonciateur du discours théorique doit conjuguer cette contrainte avec celle d'un positionnement repérable dans le champ discursif correspondant à la communauté disciplinaire de laquelle relèvent ses propositions théoriques. De même que l'effacement énonciatif dans le discours journalistique est produit dans la tension entre l'exigence de neutralité et l'impossibilité de ne pas évaluer, l'effacement énonciatif dans le discours théorique répond au paradoxe d'un positionnement qui ne peut s'affirmer sur le mode subjectif. Les travaux de Dominique Maingueneau et de Frédéric Cossutta ont bien exploré les stratégies disponibles pour affronter ce paradoxe, essentiellement à partir de l'exemple du discours philosophique. Deux grandes voies méthodologiques peuvent être dégagées pour rendre compte de ces stratégies. 
8 La première consiste à privilégier l'examen du tissu discursif en postulant, comme le fait Frédéric Cossutta (2004: $\$ 2)$, l'interdépendance des schèmes explicatifs et des schèmes d'exposition. L'analyste traduit ainsi telle doctrine philosophique en un travail réflexif pour neutraliser " la relativité du point de vue » à partir duquel elle s'énonce. Une telle démarche présente des intérêts évidents, mais à nos yeux elle n'évite pas le risque de reconduire simplement les oppositions en cours entre les grands systèmes philosophiques, en déplaçant ces oppositions sur le plan énonciatif.

9 Selon une autre approche, mise en œuvre récemment par Johannes Angermüller, on peut à l'inverse chercher à «montrer la façon dont les textes théoriques sont lus et inscrits dans leur champ de production symbolique» (Angermüller 2009: 175), en subordonnant cette fois la lecture du discours théorique à la sociologie du champ intellectuel. Centré sur les théoriciens français des années 1960, le travail d'Angermüller consiste à identifier dans les textes la "panoplie de savoirs » et les renvois implicites qui permettent au "lecteur initié » de situer les acteurs de cette abstraite Théorie les uns par rapport aux autres. Le projet ne manque pas de séduire, en ce qu'il désacralise largement une période dorée de l'intelligentsia parisienne et fait prendre conscience des déterminations institutionnelles qui pesèrent sur ce qu'on a parfois tendance à considérer comme une éclosion spontanée du Savoir. Cela dit, le risque ici couru est de rabattre cette fois le plan du discours sur celui du champ et de se borner à un travail d'identification des références cachées.

10 Dominique Maingueneau a tenté d'identifier une voie médiane entre ces deux perspectives sur le discours théorique, en recourant notamment à la notion de « scène d'énonciation »: "le lecteur s'incorpore à un monde que configure une énonciation portée par un certain ethos, celui d'un locuteur qui s'en porte garant à travers sa manière de dire » (Maingueneau 2005 : § 27). Centrée sur le discours de Louis Althusser, l'analyse de Maingueneau dans cet article prend en compte la configuration sociohistorique propre à l'émergence de ce discours. Cette configuration intervient en effet dans la manière dont la scène d'énonciation mobilise telle communauté de coénonciateurs, tout en visant à construire telle autre.

11 On voit donc ici que l'articulation du sociologique et du discursif s'opère par un autre biais que celui offert par la question de la "prise de position » qu'Amossy et Koren plaçaient au centre d'une démarche fondée sur l'éclairage réciproque de la linguistique de l'énonciation et de la sociologie des champs, en se proposant d'examiner « comment une prise de position s'opère dans un discours qui tend à effacer les marques de subjectivité » (Amossy \& Koren 2005: §13). Tout en se situant dans ce cadre de réflexion, le présent article entend explorer d'autres manières d'articuler le sociologique et le discursif, en défendant l'idée qu'un discours peut argumenter moins pour prendre position dans un état donné d'un champ, que pour proposer un nouvel espace de positionnements, pour configurer de nouveaux cadres formels, représentationnels et institutionnels à partir des obsessions ou des attentes d'une communauté. La démarche se veut donc bien rhétorique, mais fondée sur l'hypothèse que les opérations rhétoriques prennent en écharpe des phénomènes sociologiques et que la matérialité des discours analysés trouve sa genèse dans la sémiosis sociale qui garde trace de ces phénomènes et qui confère un sens particulier aux stratégies mises en évidence par l'analyse discursive.

12 En effet, s'il est indéniablement lié au concept d'ethos et à une démarche de persuasion, l'effacement énonciatif a également affaire avec la doxa. Parmi les marques de 
"l'appareil formel de l'effacement énonciatif», Alain Rabatel range "les énoncés doxiques" (Rabatel 2004), là où Robert Vion parlait de "parallélisme » pour caractériser les mises en scène énonciatives permettant « au locuteur de construire son propos en relation avec d'autres opinions » (Vion 2001 : 333). Nous rejoignons ici l'idée, formulée par Maingueneau et citée plus haut, de "communautés de co-énonciateurs » impliquées dans la scène énonciative d'un discours d'où l'énonciateur semble absent. La convocation de ces communautés pourrait relever de ce que nous avons appelé plus haut le « contrat rhétorique » passé entre les acteurs de l'échange discursif. De manière plus ou moins explicite, l'énonciateur du discours théorique s'engage sur l'extension doxique impliquée par son effacement ; autrement dit, l'absence de marquage déictique et axiologique étant codée par l'inscription dans le genre dit «de la théorie », cette absence est compensée par des indices (paratextuels notamment : voir le rôle joué par les titres, intertitres, notes et références) qui renseignent sur l'ampleur de la reconfiguration doxique à laquelle devra se prêter l'énonciataire.

Notre conception de la doxa se nourrit principalement des considérations de Ruth Amossy sur ce sujet (Amossy 2002, Amossy 2006 [2000] : 99-126). Selon elle, « les notions d'élément doxique et d'interdiscours permettent [...] de marquer à quel point l'échange argumentatif est tributaire d'un savoir partagé et d'un espace discursif, tout en évitant de conférer à ces matériaux préexistants une trop grande systématicité » (Amossy 2006 [2000] : 110). Nous utiliserons quant à nous l'expression « filière doxique » pour rendre compte d'une hétérogénéité de représentations qui se laisse cependant organiser en grands domaines (thématiques, disciplinaires, idéologiques, etc.) de savoirs partagés par des communautés d'extensions différentes. En ce sens, la doxa constitue la zone de chantier que se donne le discours dans son déploiement argumentatif. Si les éléments doxiques préexistent à ce discours, c'est surtout en tant que repères dans l'encyclopédie collective, signaux indiquant le stock de représentations qui vont faire l'objet du traitement rhétorique et axiologique. Ce traitement produit alors un nouvel agrégat d'éléments doxiques, plus ou moins bien situé dans telle portion de l'encyclopédie collective et plus ou moins bien ciblé sur telle gamme d'usagers de cette encyclopédie. La doxa n'est donc pas forcément ce dont le théoricien doit se démarquer dans son discours, mais plutôt l'ancrage qu'il donne à son argumentation. Cet ancrage est autant discursif que socioculturel : la gamme de présupposés, de représentations et de jugements qu'il mobilise renvoie en effet autant à des cadres formels de mise en discours qu'à des institutions, des communautés de lecteurs, des référents, des axiologies qui ne sont lisibles qu'en fonction d'une certaine époque ou d'un certain état de société. C'est le propre d'un contrat rhétorique que d'assurer l'ajustement de la dimension argumentative du discours avec les filières doxiques qui constituent à la fois son substrat socioculturel, son matériau rhétorique et son produit fini rendu à la circulation sociale.

Le secteur particulier de la théorie littéraire offre de nombreux exemples de ce type de contrat rhétorique, où l'énonciateur donne à ses jugements de valeur les apparences du jugement de fait (sur tel texte, tel auteur, telle méthode), tout en contrôlant les effets de ces jugements par les appels à telle communauté de co-énonciateurs, qui partagent telle gamme de références, de systèmes de valeurs et qui relèvent de telle configuration socio-historique. Dans la suite de cet article, nous allons nous attacher à un cas d'effacement énonciatif dans un discours de théorie littéraire reposant sur un contrat rhétorique pour le moins problématique et engageant dès lors des rapports non maîtrisés avec la doxa. Les années dites «structuralistes » furent en effet, en France, le 
moment d'une grande redéfinition de la rhétorique métalittéraire traditionnelle, caractérisée jusqu'alors par des approches jugées trop "historiques" ou trop «subjectives" de la littérature. L'énonciateur métalittéraire structuraliste s'abstient désormais de situer son point de vue, ce qui donne l'occasion à une toute nouvelle classe d'intellectuels de tenir sur la littérature un discours qui était jusqu'alors proprement inaudible. Or, ce novum ${ }^{3}$ présente la particularité de s'afficher comme transcendant les divisions discursives traditionnelles: l'effacement énonciatif ne s'inscrit pas dans un contrat rhétorique précis, mais est l'instrument d'un brouillage des filières doxiques, d'une superposition des dimensions axiologiques (implicites) du discours et, partant, d'une extension maximale de ses conditions d'historicité. Nous évoquerons notamment les effets particuliers que cet effacement énonciatif a sur la représentation de la littérature et sur les manières de se dire écrivain dans la France des années 1960.

\section{Le cas Kristeva} de Julia Kristeva, souvent considérée comme l'une des figures les plus emblématiques de la French Theory. Plusieurs indices font en effet de cette auteure une sorte d'exception paradigmatique dans la production théorique structuraliste et poststructuraliste. Une exception qu'il nous a paru d'autant plus intéressant d'observer de plus près que ses écrits sont nimbés du mythe de l'obscurité fascinante. Plusieurs témoignages font en effet état soit d'un éblouissement intellectuel total, soit d'une incompréhension agacée, soit enfin d'un scepticisme prudent ou rageur, face aux textes de la théoricienne dont les effets d'ethos furent sans doute les plus sensibles ${ }^{4}$.

Plus objectivement, Kristeva apparaît comme une exception paradigmatique par son étonnante trajectoire ${ }^{5}$, qui la conduit de Sofia à Paris, où elle débarque au milieu des années 1960 alors qu'elle n'a pas 25 ans, en tant que boursière du gouvernement français. Elle arrive avec un solide bagage intellectuel sur le formalisme russe et parvient immédiatement à faire valoir ce précieux capital culturel et sa position d'extériorité pour imprimer une influence majeure dans les milieux structuralistes parisiens. Au séminaire de Barthes, elle introduit l'œuvre de Mikhaïl Bakhtine et contribue aussitôt à réorienter le structuralisme français grâce aux concepts d'intertextualité et de dialogisme. Elle fréquente Lévi-Strauss et Lacan, se lie avec Benveniste, est intégrée dans l'équipe de Tel Quel, alors à la pointe de l'avant-garde intellectuelle. Bref, elle s'impose avec une rapidité étonnante comme la nouvelle étoile de ce milieu bouillonnant et comme celle par qui arrive la révélation unifiante de toutes les voies nouvelles de l'intelligence: le formalisme, l'intertextualité, le sujet désirant, le langage poétique. Cette fascination qu'elle exerça sur ses contemporains se transposera quelques années plus tard aux États-Unis, où son discours connaît une efficace extraordinaire, notamment par le biais de l'étiquette déformante et globalisante de French theory 6 .

17 En somme, par ces différents indices, il nous a semblé que Kristeva pouvait être un beau cas d'école: mythifiée par ses contemporains et, encore aujourd'hui, cible des polémistes, elle réalise par ses multiples casquettes (sémioticienne, psychanalyste, romancière, critique littéraire, linguiste) le projet de fusion disciplinaire du structuralisme à son plus haut degré de généralité; elle témoigne en outre du rôle 
central joué par la littérature dans cette nouvelle conception du savoir et incarne l'exemple parfait de la circulation internationale du paradigme structuraliste (au sens large), des pays de l'Est vers la France, puis vers les États-Unis, avec tous les effets de déplacement qu'entraînent de telles circulations.

Par son exceptionnalité paradigmatique, le cas Kristeva nous semble également désigner de manière précise la particularité du rapport problématique qu'entretient à cette époque le discours théorique avec la doxa : quant à son expression, il est pris dans la contradiction entre l'idéal de démocratisation et l'entretien d'une forme d'hermétisme élitiste; quant à son contenu, il hésite entre un apolitisme purement spéculatif et un engagement en fonction d'idéaux révolutionnaires. Ces hésitations et contradictions d'époque ne permettent pas de garantir un contrat rhétorique stable, dans lequel trouverait sa place la stratégie de l'effacement énonciatif. Dès lors, quels statuts et quelles fonctions assume une telle stratégie, dans le cadre d'un discours théorique sur la littérature? C'est à cette question que nous allons à présent nous attacher, à partir de trois des plus fameux textes de Kristeva : «Pour une sémiologie des paragrammes ", l'article le plus hermétique (et donc polémique) de Kristeva, paru dès 1966 dans Tel Quel, puis republié en 1969 dans l'ouvrage Semeiotikè (Kristeva 1969, dorénavant abrégé PSP) ; «La sémiologie : science critique et/ou critique de la science » (Kristeva 1968a, dorénavant abrégé SSC) et "Problèmes de la structuration du texte » (Kristeva 1968b, dorénavant abrégé PST), tous deux parus dans La Nouvelle Critique, puis repris dans la fameuse Théorie d'ensemble de Tel Quel, parue en 1968.

\section{Trois opérations graduelles autour de la doxa}

L'effacement énonciatif peut être ici observé à la faveur de trois opérations graduelles autour de la doxa: a) des mises en concurrence; b) des connexions intra- et interdiscursives ; c) des déplacements et recouvrements. Dans tous les cas, le locuteur fait mine de neutraliser sa deixis et sa subjectivité modale ou axiologique pour apparaître en sur-énonciateur ${ }^{7}$ désincarné, grand ordonnateur des nouvelles représentations qui s'imposent à une multiplicité de communautés de co-énonciateurs.

Par mises en concurrence doxiques, nous désignons la confrontation, non plus d'un savoir théorique avec les évidences d'un sens commun local, mais de plusieurs filières d'opinions entre elles. Ces filières relèvent autant de l'état des lieux d'une discipline que d'un savoir partagé sur la littérature ou sur la société, d'un discours marxiste constitué ou d'un système de notations importé des mathématiques. Toutes ces filières, de dimensions variables, sont en coprésence dans le discours, simultanément mises en scène et évaluées par le théoricien, dont le point de vue - bien que lisible à chaque fois en creux dans l'implicite des termes choisis - ne peut être reconduit à une position globale cohérente, situable dans un champ polémique bien délimité. Par exemple, Kristeva évoque à plusieurs reprises le «structuralisme actuel », les "défauts » qu'il convient de reconnaître (PSP : 203) et ceux « qu'on croit être [...] inhérents » (PSP : 174) à ce paradigme (nous soulignons). Ailleurs, c'est la «théorie linguistique » qui est dite «difficilement sensible à la violence de la langue » (PSP : 204), tandis que «comme on le sait, dans le modèle chomskien [sic] la transformation est donnée d'avance arbitrairement dans un nombre fini de règles » (PST : 301) (nous soulignons).

21 Toujours sur un plan a priori disciplinaire, mais à propos de la sémiologie cette fois, l'auteure évoque «l'hostilité mal camouflée de la parole (de la "conscience") bourgeoise 
dans ses multiples variantes [...] qui déclarent cette recherche "obscure", "gratuite", "schématique" ou "appauvrissante" » (SSC : 80). On a donc ici affaire à un élargissement conséquent du champ polémique concerné, puisque c'est la doxa «bourgeoise » dans son ensemble qui est convoquée. Parfois, l'indétermination doxique est encore majeure, comme lorsque l'auteure affirme que «dans notre société le mot est devenu clarification, pétrification, carcan» et que, selon cette conception, "[o]n s'étonne [...] si chez quelqu'un il brasse des espaces et attire les vibrations qui décrivent un rythme » (PSP : 204, nous soulignons).

De manière privilégiée, ces confrontations doxiques (d'étonnements, de croyances, etc.) portent sur la littérature et c'est souvent une doxa métalittéraire (plus ou moins supportée par un savoir formalisé) qui est jouée contre une autre (supportée par un autre type de formalisation), comme dans cet extrait :

La notion du langage poétique [telle que rationalisée par la science linguistique] comme déviation du langage normal [...] a remplacé la conception naturaliste de la littérature comme reflet (expression) de la réalité, et cette notion est en train de se figer en un poncif qui empêche d'étudier la morphologie proprement poétique (PSP : 177).

La parole théorique et sa justification se tissent ainsi au fil d'une intrication serrée entre de multiples autres discours : ce que l'auteure appelle « le discours dit quotidien » (PSP : 176) et sa "conception générale du texte littéraire " (PSP : 175) - ou «ce que notre culture appelle "la littérature" » (PST : 302) -, les croyances quant aux carences et aux renouvellements possibles du structuralisme et de la linguistique, les conceptions de Marx, de Lénine, aussi bien que de Derrida, sur ce qu'est la démarche scientifique par rapport à la société (PSP : 205), mais aussi les évidences formulées par les axiomes et dans la langue formalisée des théorèmes de la théorie mathématique des ensembles, comme dans cet énoncé par exemple : «Le théorème généralisé de l'existence postule, on le sait, que : [...] » (PSP : 189, nous soulignons).

Autrement dit, ce qui est mis en scène ici, c'est une série de savoirs partagés par des communautés d'extensions très diverses (ce que nous appelons des filières doxiques). L'interconnexion de ces savoirs et croyances confère au discours un champ de pertinence très étendu, puisque, en l'absence d'un contrat rhétorique qui permettrait au destinataire de reconstituer les rapports d'assomption doxique pertinents, les secteurs de connaissances que le discours fait s'entrechoquer se sur-impriment les uns aux autres.

Cette sur-impression est précisément la deuxième opération à observer pour évaluer le fonctionnement de l'effacement énonciatif dans ce discours. En convoquant plusieurs filières doxiques, l'énonciateur s'octroie également le rôle d'opérer les connexions intra- et interdiscursives qui situent ces filières dans une dramaturgie spéculative plus globale. Les plus évidentes de ces connexions sont celles qui configurent une temporalité interne au discours, en se référant au rythme de déploiement de la parole théorique : «nous venons de constater ", « rappelons ", « insistons " pointent bien vers une source énonciative, mais qui n'est que le support vide des prédicats qui jalonnent le texte en différents moments. Du même ordre sont les périphrases qui thématisent l'acte de convocation doxique évoqué plus haut, comme lorsque Kristeva écrit: "Soulignons entre parenthèses que Marx insiste sur la relativité et l'historicité de la valeur et surtout de la valeur d'échange » (SSC: 88 ), ou « notons l'apport magistral de Husserl et d'Heidegger, mais surtout de Freud» (SSC: 89), ou encore «À cette 
conception du modèle génératif nous ajouterons les modifications introduites par la linguistique soviétique [...]» (PST : 300).

Les autres soudures discursives fortement thématisées sont celles qui concernent les relations d'implication et de présupposition que les unités du discours entretiennent entre elles. Les "donc », " ainsi », " autrement dit », «implique que », " exige que », "nécessairement » émaillent le discours de Kristeva et balisent fermement un parcours logique entre les différentes filières doxiques. Les contraintes selon lesquelles se dessine ce parcours ne sont jamais aussi nettes que lorsqu'elles apparaissent sous la forme d'une relation d'exclusivité, comme dans cet exemple (parmi tant d'autres), où l'auteure présente les théorèmes d'existence des mathématiques des ensembles comme fournisseurs de « concepts permettant de poser de façon nouvelle et sans eux impossible, l'objet qui nous intéresse : le langage poétique" (PSP : 189, nous soulignons). Les exemples sont nombreux où les tournures en "ne ... que " imposent l'évidence d'une architecture conceptuelle (au sens large) à sens unique, comme lorsque l'auteure définit le livre lisible comme une fermeture ouverte sur l'infini : «Cette lisibilité du fermé ouvrant vers l'infini n'est accessible complètement qu'à celui qui écrit [...] 》 (PSP : 180).

Nous pourrions évoquer encore d'autres formes de connexions par lesquelles le discours consolide et thématise sa propre cohérence et sa solidarité avec un vaste ensemble de filières doxiques: pensons aux gestes de glose, d'allusion, de mise en exergue, de comparaison, de définition terminologique, qui sont légion dans les textes de Kristeva, et qu'il serait fastidieux de détailler ici ${ }^{8}$.

Abordons à présent la troisième opération rhétorique, qui parachève en quelque sorte celles de mises en concurrence et de connexions que nous venons d'évoquer : il s'agit des déplacements et recouvrements que le discours opère dans l'encyclopédie générale qu'il mobilise. Ces déplacements encyclopédiques touchent essentiellement à deux unités: le langage poétique et l'opération de théorisation elle-même. Le langage poétique est totalement débranché de tous les préjugés qui orientaient son appréhension «esthétique »: le sens comme totalité représentable, la poésie comme écart, etc. L'unité ainsi débarrassée de ses attaches doxiques est prédiquée selon une nouvelle évidence, celle d'une nécessaire isomorphie entre l'objet et l'appareil de description : "Il est évident que le langage poétique comme système complémentaire obéissant à une logique différente de celle de la démarche scientifique, exige, pour être décrit, un appareil qui prenne en considération les caractéristiques de cette logique poétique » (PSP : 176). Ce déplacement du «langage poétique » de la doxa esthétique vers la nécessité théorique a des conséquences évidentes sur la conception de la pratique littéraire, conçue comme "exploration et découverte des possibilités du langage » (PSP : 178) et induit la confusion entre la démarche du linguiste et celle de l'écrivain, désormais tous deux unis dans la même recherche sur le «devenir des significations des signes » (PSP : 179). Cette nouvelle doxa du langage poétique résorbe également les oppositions entre le fini et l'infini, entre l'écriture et la lecture, entre la pratique d'écriture des sujets historiques que sont par exemple les écrivains Lautréamont et Mallarmé, et la tâche descriptive du sujet absolu qu'est «le sémioticien", puisque tous sont pris dans le même paradigme de la "recherche poétique ».

En outre, à la faveur de ce déplacement, le langage poétique se voit doté d'un statut d'exceptionnalité que souligne le transcodage mathématique dont il fait l'objet: 
observé par le prisme de la " "numérologie" paragrammatique » (PSP : 193), il acquiert une valeur hautement singulière, puisque "[l]e paragramme est le seul espace du langage où le I ne fonctionne pas comme unité, mais comme entier, comme tout, parce qu'il est double » (PSP : 194).

Cette exceptionnalité très générale peut dès lors suivre un mouvement inverse et se reformuler dans des termes cette fois plus proches du sens commun, notamment selon l'opposition audace vs conservatisme, et consolider son évidence en s'appuyant sur un panthéon bien identifiable. L'extrait suivant est très révélateur de ce retour de vernis doxique et de polarités axiologiques communes sur l'objet de la théorie :

L'écriture qui a l'audace de suivre le trajet complet de ce mouvement dialogique que nous venons de représenter par le tétralemme [...] n'appartient pas à ce que l'on appelle traditionnellement « littérature » [...]. L'écriture paragrammatique est une réflexion continue, une contestation écrite du code, de la loi et de soi-même [...]. L'écriture de Dante, Sade, Lautréamont en est un exemple dans la tradition européenne (PSP : 197).

De manière tout à fait symétrique, le geste de théorisation lui-même suit un trajet semblable, d'arrachement à son encyclopédie d'origine, de reformulation selon un nouveau système de valeurs et de recouvrement par des grilles de compréhension déjà disponibles dans le sens commun. L'acte de théoriser est ainsi d'abord dirigé vers le cadre disciplinaire qui est censé en être le support : alors que la conception commune des autres sciences veut que la théorie soit un outil pour penser les objets de ces sciences, la sémiotique se conçoit quant à elle comme « un type de pensée où la science se vit (est consciente) du fait qu'elle est une théorie » (SSC : 82). La théorie est ainsi posée comme principe constitutif de la discipline; celle-ci est théorie et se conçoit comme telle dans un retour réflexif permanent. On assiste sans doute là à l'un des déplacements encyclopédiques majeurs de ce type de discours, qui achève de rendre éminemment problématique le statut de sa source énonciative : si c'est le propre de la discipline sémiotique de se penser comme théorie, où est donc située la parole qui pense ce retour réflexif lui-même?

Toujours est-il que, comme pour "le langage poétique», ce déplacement de "l'opération théorique " s'accompagne d'une reformulation selon les termes de la lutte idéologique et de la contestation politique : "Confrontant les découvertes actuelles des métamathématiques et de la logique mathématique avec les structures du langage poétique moderne, la sémiotique [...] occupera une position idéologique-clé dans un processus globalement révolutionnaire» (PSP : 207). La reformulation adopte également des termes métaphoriques de portée plus large encore, qui situent la démarche évoquée dans une gamme de pratiques associées à un ethos facilement identifiable: "l'axiomatisation paragrammatique est une démarche fougueuse, "exagérée" et "excentrique" » (PSP : 206).

Tous ces déplacements et reformulations produisent finalement une nouvelle totalité doxique cohérente, selon laquelle "le langage poétique» est dissocié de «la littérature » pour être versé dans une pratique de « recherche ", à laquelle s'associent la discipline sémiotique et sa propre théorisation. Cette pratique se conçoit selon une éthique contestataire, risquée et exploratoire et est inscrite dans une histoire très sélective, qui retient quelques figures littéraires d'exceptions en diachronie (Dante, Sade, Lautréamont, Mallarmé) et les pose comme symptômes d'un processus qui se densifie dans le présent de l'énonciation: «Un nouvel idéologème est en train de se 
constituer à partir du $20^{\mathrm{e}}$ siècle avec les nouvelles structures textuelles (Mallarmé, Lautréamont) et qui continuent [sic] à se chercher aujourd'hui » (PST : 316).

Quelles conséquences peut-on tirer de ces différentes opérations rhétoriques quant à l'ancrage axiologique assumé par l'énonciateur caché au creux de ce discours et, partant, quant à son embrayage historique ? L'effacement de l'énonciateur derrière ses manœuvres de reconfiguration doxique permet que les valeurs soient présentées sur le mode normatif et éthique : sans support subjectif affirmé, elles acquièrent l'évidence et le statut prescriptif d'un code de conduite généralisé à l'ensemble des objets brassés par le discours. Ainsi, par exemple, de la fonction de dévoilement assignée au recours aux mathématiques, qui « aidera à ramener à la conscience de tout utilisateur du code le concept de l'infinité du langage poétique » (PSP : 200, nous soulignons), ou encore qui permettra de " révéler que tout art est une science appliquée » (PSP : 204).

Ces nouvelles évidences affichent tout particulièrement leur statut normatif lorsqu'elles s'appliquent au canon littéraire, pour en disqualifier des pans entiers et en proposer une nouvelle hiérarchie, en fonction de nouvelles valeurs. Le passage suivant est très révélateur de l'application normative des nouvelles évidences et donc du glissement implicite du jugement de valeur au jugement de fait :

Tous les codes poétiques qui se limitent à postuler uniquement une fonction $\varphi\left(x_{1} \cdots\right.$ $\left.x_{\mathrm{n}}\right)$ sans réaliser le théorème de l'existence, c'est-à-dire sans se construire de séquences équivalentes à $\varphi$, est un [sic]code poétique raté. Ceci explique entre autres le fait que l'échec de la littérature " existentialiste » [...] est lisible d'une façon incontestable dans son style métaphysique et son incompréhension totale du fonctionnement du langage poétique.

Lautréamont était un des premiers à pratiquer consciemment ce théorème. (PSP : 190 , nous soulignons ${ }^{9}$ ).

Cet extrait nous montre à quel point ces opérations rhétoriques orientent une conception de l'écrivain et des manières de se dire écrivain tout à fait précises et valorisées selon une axiologie tout aussi élitiste que celle qui est dénoncée pour sa dépendance avec l'ordre social bourgeois et marchand. Sur le modèle de Philippe Sollers, le véritable écrivain doit se dire chercheur, renoncer consciemment à la conception du sens comme totalité représentable et concevoir son œuvre comme l'exploration formalisée de l'infinité des possibles du langage, selon une éthique antihumaniste de la dés-illusion permanente.

Ces conceptions sont évidemment très situées socio-historiquement, mais cette situation n'est pas déductible de ce que le discours donne à lire. En effet, lorsqu'il rebranche ses unités sur un fond doxique, le texte évoque bien «la société bourgeoise ", ou " notre culture ", " notre horizon intellectuel », mais ces mentions ne fonctionnent pas vraiment comme des embrayeurs qui permettraient de situer le sujet de l'énonciation. Celui-ci met en place, et de manière beaucoup plus nette, une temporalité parallèle, qui est celle du déploiement théorique lui-même et qui englobe les «nous" faussement inclusifs évoqués plus haut. La "recherche» mise en scène s'étend universellement et intemporellement des textes de l'Antiquité chinoise jusqu'au roman Drame de Philippe Sollers, publié en 1965, et la dialectique qui s'opère contre « le texte social bourgeois » n'est qu'un moment d'une histoire ainsi conçue sur le mode téléologique et située au-delà de tout point de vue subjectivé. 


\section{Deux formes de subjectivation rétrospective}

38 Ce que nous venons de montrer dans les textes des années 1960 peut être mis en contraste avec deux autres interventions de Julia Kristeva, plus tardives, qui manifestent deux formes de subjectivation rétrospective des discours théoriques. Tandis que, dans les articles analysés, l'effacement énonciatif s'opérait à la faveur d'une nouvelle doxa totalisante, quelques décennies plus tard, Kristeva reformule les grandes tendances de sa période structuraliste selon des contrats rhétoriques beaucoup plus nets : d'une part les contributions aux actes du colloque De Tel Quel à l'Infini, qui eut lieu à Londres et à Paris en 1995 (l'une sous forme d'article [Kristeva 2000a], l'autre sous forme d'entretien [Kristeva 2000b]); d'autre part les propos échangés en 1982 avec Pierre Encrevé et Jean-Claude Chevalier à propos de la constitution du champ disciplinaire de la linguistique, publiés dans le volume Combats pour la linguistique (Chevalier \& Encrevé 2006 : 263-296).

Dans les deux cas, le contrat rhétorique est clairement identifié : sorte de muséification participante d'une avant-garde littéraire d'un côté, témoignage pour une histoire sociale de la linguistique de l'autre. Chacun de ces contrats autorise, voire favorise, le recours à une énonciation fortement subjectivée. Ainsi, dans les actes du colloque de 1995, Kristeva reformule son programme de recherches en s'identifiant comme « femme » et « migrante " ${ }^{10}$, inscrite dans une collectivité historiquement située, qu'elle caractérise par la métaphore des « samouraïs » :

Tel Quel reste aujourd'hui encore pour moi une complicité réservée, l'effervescence invisible. Pendant que s'installait la société postindustrielle qui standardise et banalise, nous prenions le risque d'une pensée difficile que certains trouvaient ésotérique. Mais, pour nous, c'était l'évidence, nous n'y voyions aucun risque. En ce sens, nous étions des samouraïs (Kristeva 2000a : 33).

On voit bien dans cet extrait de quelle manière se reformule la mise en concurrence doxique analysée plus haut : elle est ici supportée par un " nous » très situé, dans lequel s'inclut l'énonciatrice. La normativité qui découlait du discours théorique est ici endossée par cette seule collectivité, située dans l'évidence métaphorique des "samouraïs". Cette métaphore, ainsi que la caractérisation spécifique de Kristeva comme femme émigrée, s'accordent avec l'entreprise de muséification participante de l'avant-garde Tel Quel, qui fonde le contrat rhétorique du discours du collectif.

41 Tout autre est le contrat qui sous-tend le discours de Kristeva dans les entretiens avec Pierre Encrevé et Jean-Claude Chevalier. La subjectivation rétrospective s'opère ici selon d'autres pôles d'identification, qui prennent sens en fonction de la perspective d'histoire sociale de la linguistique adoptée par les deux intervieweurs. Kristeva s'y situe dans des «nous » à dimensions variables (Tel Quel, « le milieu étudiant », «notre génération », « Mai 68 ») qui personnifient la rumeur intellectualisante de l'époque, et construit sa singularité sur le mode de la "marginalité ». Elle présente par exemple son goût pour la théorie comme une spécialité exotique aux yeux des linguistes français de l'époque ${ }^{11}$, associe sa vocation interdisciplinaire à son " destin d'étrangère " (Chevalier \& Encrevé 2006: 279) et, surtout, met en scène ses réactions très personnelles aux questions institutionnelles et aux aspects interpersonnels qui animaient le champ linguistique de l'époque. Voir, par exemple, à propos de Greimas :

Moi aussi [avec Lévi-Strauss], je trouvais cette approche d'un dépouillement logique que les Français ignorent (Rires). Trop arbitraire. Du Hjelmslev passé à la moulinette et qui ne pouvait pas embrayer sur la réalité littéraire. Et pourtant lui, Greimas, il 
prétendait rendre compte des textes littéraires, Rimbaud, entre autres (Chevalier \&

Encrevé 2006 : 274). reformulées sur le mode subjectif explicite («j'envisage », «je pose », «j'appelle», « ma thèse ", « hypothèses que j'ai formulées à ma façon »), dans les termes d'une conviction personnelle et non plus comme un discours de vérité sur le monde. Plutôt que "théoricienne », Kristeva s'affiche comme "sémiologue et psychanalyste », dont la marginalité dans le champ linguistique français des années 1960 à 1980 se donne désormais à voir aussi sous l'étiquette du " plaisir » et du « ludisme ", plutôt que sous celle de l'hermétisme formaliste et terroriste ${ }^{12}$. Il est évident que, dans la galerie de portraits proposée par Encrevé et Chevalier (où, à part Kristeva, il n'y a que des hommes, aussi sévères que Pottier, Martinet, Dubois ou Greimas), la Bulgare occupe la place de la belle étrangère, dont l'impertinence et la marginalité sont tolérées au nom de la séduction pour l'audace et l'indépendance d'esprit, pour former ce tableau vivant que constitue Combats pour la linguistique.

\section{Retour sur la méthode}

43 L'examen de ces deux formes de subjectivation rétrospective du discours théorique nous introduit à la partie finale de cet article, qui entend poursuivre l'interrogation méthodologique ouverte dans nos premières pages. L'effacement énonciatif peut être analysé en tant qu'il participe à la construction d'un certain ethos, lui-même pris dans une stratégie argumentative orientée par une prise de position de l'agent dans son champ d'activité. Cela dit, si l'analyse rhétorique est toujours forcément sociologique, c'est aussi en ce qu'elle interroge la genèse et l'efficace des discours en prenant en compte les cadres formels, représentationnels et institutionnels partagés par une communauté discursive. Ces cadres ne sont pas des données préalables à la disposition de l'analyste pour " contextualiser » les discours, mais constituent le matériau même du travail rhétorique, dont le protocole peut être plus ou moins codifié dans un contrat entre partenaires de l'échange discursif. En l'occurrence, l'effacement énonciatif à l'œuvre dans le discours théorique est le plus souvent réglé à l'avance et admis comme tel par des énonciataires capables de situer malgré tout la source énonciative et dès lors de limiter la portée persuasive de son discours. Or, le discours de Julia Kristeva - saisi ici comme symptomatique de l'effet-théorie des années 1960 - efface l'énonciateur en profilant une nébuleuse de filières doxiques, qui renvoient à autant de cadres formels, représentationnels et institutionnels mis à mal ou renégociés.

44

Il n'empêche que moins l'on sait de quoi on parle, plus on identifie qui parle, et pour qui : les discours les plus désubjectivés de Kristeva ont conduit immédiatement à la starification de leur locutrice, par une communauté de «lecteurs-de-Kristeva » qui ne préexistait pas à la profération de ce discours. Non réglée par contrat préalable, la question de l'auditoire (de l'existence d'un auditoire à même de recevoir le discours) constitue ainsi l'un des enjeux mêmes de l'entreprise de persuasion de ce type de discours. Les bouleversements sociologiques (démographiques, économiques, symboliques, etc.) que connaît la France des années 1960 expliquent en partie la pertinence d'un tel enjeu et le succès de discours qui s'y sont confrontés avec le plus de radicalité. Mais il faut sans doute également émettre l'hypothèse, avec Kristin Ross, que l'ensemble du discours social de la France gaullienne préparait une place centrale à ce

Argumentation et Analyse du Discours, 5 | 2010 
type de discours: idéal de technicité, effacement du sujet de l'histoire, déni de la temporalité historique, redéfinition de la spécificité de «l'esprit français » et des effets de légitimité qui lui sont associés (notamment via la littérature et l'image de l'écrivain), sont en effet quelques-uns des principaux complexes discursifs en circulation dans l' épistémè de l'époque, marquée par les présupposés idéologiques de la mort de l'Homme et de la fuite hors de l'Histoire (Ross $2006: 221-276$ ).

La stratégie de l'effacement énonciatif gagne ainsi à être envisagée dans le cadre élargi d'une configuration socio-historique et socio-discursive, qui en conditionne la lisibilité et l'efficace. La notion de contrat rhétorique entend contribuer à l'élaboration conceptuelle d'un tel cadre : défini comme le consensus préalable, entre énonciateur et énonciataire, quant à l'extension (par exemple, telle portion de l'univers de croyances) et aux modalités (par exemple, visée vs dimension argumentative, effacement énonciatif, narration, etc.) du projet de persuasion du discours, ce contrat a) participe le plus souvent de contraintes de genres, b) est gradué du plus explicite au plus implicite, et du mieux au moins bien respecté, c) peut favoriser l'émergence d'une communauté discursive, se reconnaissant dans tel type de contrat.

Si l'analyse de tels contrats peut être qualifiée de socio-rhétorique, c'est donc qu'elle se veut attentive à la reconfiguration symbolique et institutionnelle des espaces où ils sont établis, autant qu'aux déterminations externes de ces contrats ou qu'aux prises de position qu'ils permettent d'exprimer de manière plus ou moins contournée.

\section{BIBLIOGRAPHIE}

Amossy, Ruth. 2002. « How To Do Things with Doxa : Toward an Analysis of Argumentation in Discourse », Poetics Today $23: 3$, « Doxa and Discourse. How Common Knowledge Works », 464-487 Amossy, Ruth. 2006 [2000]. L'argumentation dans le discours, $2^{\mathrm{e}}$ édition (Paris : Armand Colin) Angenot, Marc. 1989. 1889. Un état du discours social (Québec : Le Préambule) Angermüller, Johannes. 2009. «Citer les autorités du discours intellectuel. Tel Quel et la création de la Théorie ", Regards sociologiques 37-38, 175-183

Brandt, Joan. 1989. « The systematics of a non-system : Julia Kristeva's revisionary semiotics », The American Journal of Semiotics $5: 1,133-150$

Chevalier, Jean-Claude \& Pierre Encrevé. 2006. Combats pour la linguistique, de Martinet à Kristeva. Essai de dramaturgie épistémologique (Paris : ENS)

Cossutta, Frédéric. 2004. « Neutralisation du point de vue et stratégies argumentatives dans le discours philosophique ", Semen [en ligne] 17, «Argumentation et prise de position : pratiques discursives ", mis en ligne le 29 avril 2007. URL : http://semen.revues.org/2321

Cusset, François. 2003. French Theory. Foucault, Derrida, Deleuze \& Cie et les mutations de la vie intellectuelle aux Etats-Unis (Paris : La Découverte)

Dosse, François. 1992. Histoire du structuralisme, 2 vol. (Paris : La Découverte) 
Fish, Stanley. 2007 [1980]. Quand lire c'est faire. L'autorité des communautés interprétatives (Paris : Les prairies ordinaires)

Gleize, Mélanie. 2005. Julia Kristeva au carrefour du littéraire et du théorique (Paris : L'Harmattan)

Kerbrat-Orecchioni, Catherine. 1978. « Déambulation en territoire aléthique », [Collectif]. Stratégies discursives. Actes du colloque du Centre de Recherches Linguistiques et Sémiologiques de Lyon 20-22 mai 1977 (Lyon : Presses universitaires de Lyon), 53-90

Kerbrat-Orecchioni, Catherine. 2009 [1980]. L'énonciation. De la subjectivité dans le langage, $4^{\mathrm{e}}$ édition (Paris : Armand Colin)

Koren, Roselyne. 1996. Les enjeux éthiques de l'écriture de presse et la mise en mots du terrorisme (Paris : L'Harmattan)

Kristeva, Julia. 1968a. « La sémiologie : science critique et/ou critique de la science », Tel Quel. Théorie d'ensemble (Paris : Seuil), 80-93

Kristeva, Julia. 1968b. « Problèmes de la structuration du texte », Tel Quel. Théorie d'ensemble (Paris : Seuil), 297-315

Kristeva, Julia. 1969 [1966]. « Pour une sémiologie des paragrammes », Kristeva, Julia. Séméiotiké. Recherches pour une sémanalyse (Paris : Seuil), 174-207

Kristeva, Julia. 2000a. « Les samouraïs tels quels », Forest, Philippe \& Patrick Ffrench (éds). De Tel Quel à L'Infini, l'avant-garde et après ?, Actes des colloques de Londres et de Paris (1995) (Nantes : Pleins feux), 21-33

Kristeva, Julia. 2000b. «Un temps d'enthousiasme serein. Entretien avec Julia Kristeva », Forest, Philippe \& Patrick Ffrench (éds). De Tel Quel à L'Infini, l'avant-garde et après ?, Actes des colloques de Londres et de Paris (1995) (Nantes : Pleins feux), 285-294

Maingueneau, Dominique. 2005. «Code langagier et scène d'énonciation philosophique », Rue Descartes $4: 50$, «L'écriture des philosophes », 22-33

Patron, Sylvie. 2000. Critique 1946-1996 : une encyclopédie de l'esprit moderne (Clamecy: Imec)

Philippe, Gilles. 2002. «L'appareil formel de l'effacement énonciatif et la pragmatique des textes sans locuteur ", Amossy, Ruth (éd.). Pragmatique et analyse des textes (Tel-Aviv : Université de TelAviv), 17-34

Rabatel, Alain. 2004. « Effacement énonciatif et effets argumentatifs indirects dans l'incipit du Mort qu'il faut de Semprun », Semen [en ligne] 17, «Argumentation et prise de position : pratiques discursives », mis en ligne le 29 avril 2007. URL : http://semen.revues.org/2334

Rabatel, Alain. 2008. Homo narrans. Pour une analyse énonciative et interactionnelle du récit, $2 \mathrm{t}$.

(Limoges : Lambert Lucas)

Ross, Kristin. 2006 [1996]. Rouler plus vite, laver plus blanc. Modernisation de la France et décolonisation au tournant des années 60 (Paris : Flammarion)

Sokal, Alan \& Jean Bricmont. 1997. Impostures intellectuelles (Paris : Odile Jacob)

Vion, Robert. 2001. «"Effacement énonciatif" et stratégies discursives », De Mattia, Monique \& André Joly (éds). De la syntaxe à la narratologie énonciative (Paris - Gap : Ophrys), 331-354 


\section{NOTES}

1. L'auteur tient à remercier vivement le comité de rédaction de la revue Argumentation et Analyse du Discours, et en particulier Ruth Amossy, pour le suivi attentif qu'ils ont accordé à cet article. Celui-ci n'aurait pu être finalisé sans leurs suggestions de lecture et leurs remarques.

2. «Un certain nombre de procédés hétérogènes (la formulation présuppositionnelle de certains éléments d'information, l'ellipse en surface de toute mention de la source Lo, l'utilisation d'un modalisateur du type "savoir") ont [...] pour fonction de produire un "effet d'objectivité" (comme on parle d'“effet de réel") » (Kerbrat-Orecchioni $1978: 66$ ).

3. Nous reprenons ici l'expression que Marc Angenot utilise dans sa théorie du discours social (Angenot 1989).

4. Cf., entre autres, Patron 2000, Cusset 2003, Dosse 1992, Chevalier \& Encrevé 2006, Brandt 1989, Gleize 2005. Cette dernière évoque notamment la célèbre polémique soulevée par la parution des Impostures intellectuelles d'Alan Sokal et Jean Bricmont (1997), dont l'une des cibles privilégiées est Julia Kristeva.

5. Cf. Dosse 1992, $1: 398-403 ; 2: 71-84$.

6. François Cusset a bien montré dans son ouvrage French Theory les opérations de déplacement qu'ont subies les textes des penseurs français une fois transposés sur les campus américains.

7. Pour rappel, sur ce concept, cf. Rabatel 2008 : 581 s.

8. Soulignons au passage que le personnel qui, dans le texte, participe à ces opérations de connexions entre filières doxiques est lui aussi hétéroclite. Le dispositif citationnel (au sens large : aussi bien les citations proprement dites que les allusions ou les références implicites) mis en place dans le discours de Kristeva place sur le même niveau de pertinence des figures comme Derrida, Marx, Hegel ou Chomsky, et les écrivains que sont Lautréamont et Mallarmé. Autrement dit, il n'y a pas d'un côté les "théoriciens » et de l'autre les " écrivains » : tous participent au même titre à la même dramaturgie spéculative placée sous le double signe de la « recherche » et de «l'écriture ».

9. Signalons au passage, à l'appui de ce que nous venons de dire, que ce type de normativité métalittéraire rendra Kristeva plutôt suspecte dans la querelle sur le canon qui anima le champ intellectuel aux États-Unis dans les années 1970 (Cusset 2003 : 184).

10. Cf., par exemple : « S'il y a quelque chose d'original dans mon travail, je crois que cela tient à mon existence tout entière : celle d'une femme intellectuelle. Née dans les Balkans durant la deuxième guerre mondiale, ayant appris le français très jeune, [...], exilée en France afin de poursuivre son travail de recherche, adoptée par une intelligentsia universaliste à l'apogée du structuralisme et du poststructuralisme, amante, épouse, mère, tentant de penser la joie et la détresse qui en tant que telles ne m'ont pas épargnée et m'ont poussée à écrire... » (Kristeva 2000b : 292).

11. Cf., par exemple, Chevalier \& Encrevé $2006: 269$.

12. Cf., par exemple: «Il s'agit d'emprunter des descriptions linguistiques ou logiques aux différents courants des sciences du langage pour essayer de proposer un système conceptuel différent, plus ludique, attentif au jeu, au plaisir, au non-sens, au mot d'esprit » (Chevalier \& Encrevé 2006 : 290). 


\section{RÉSUMÉS}

Cet article entend contribuer à la réflexion menée sur les phénomènes d'effacement énonciatif, en particulier dans le discours théorique. L'approche se veut inséparablement rhétorique et socio-historique et porte sur un corpus composé des principaux articles publiés par Julia Kristeva dans les années 1960. Ceux-ci sont contrastés avec des interventions ultérieures de la théoricienne, répondant à d'autres enjeux et stratégies. Les effets construits par l'effacement énonciatif sont envisagés comme des opérations sur la doxa, orientées par un contrat rhétorique. Cette notion, située à l'intersection du socio-historique et du discursif, se veut un outil complémentaire pour l'analyse de l'efficace des discours dans un état de société.

This paper tackles the issue of enunciative effacement, particularly in theoretical discourse. The approach is both rhetorical and socio-historical, as it focuses on texts published by Julia Kristeva in the sixties and contrasts them with more recent Kristeva speeches, corresponding to other issues and strategies. The effects brought about by enunciative effacement are considered as operations on doxa, which are oriented by a rhetorical contract. This notion, both socio-historical and rhetorical, provides a tool for analyzing the efficiency of discourses in a given state of society.

INDEX

Keywords : doxa, enunciative erasing, Kristeva (Julia), rhetorical contract, theoretical discourse Mots-clés : contrat rhétorique, discours théorique, doxa, effacement énonciatif, Kristeva (Julia)

\section{AUTEUR}

\section{FRANÇOIS PROVENZANO}

F.R.S.-FNRS, Université de Liège 